\title{
Erratum to: Earthworm burrowing activity of two non-Lumbricidae earthworm species incubated in soils with contrasting organic carbon content (Vertisol vs. Ultisol)
}

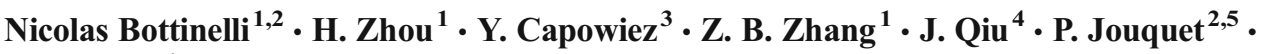 \\ X. H. Peng ${ }^{1}$
}

Published online: 25 September 2017

(C) Springer-Verlag GmbH Germany 2017

Erratum to: Biol Fertil Soils

https://doi.org/10.1007/s00374-017-1235-8

The original version of this article, unfortunately, contained errors.

The name of the author was incorrectly spelled as "X. Peng". The correct spelling is "X. H. Peng" and the corresponding author of this article was changed from Nicolas Bottinelli to $\mathrm{X}$. H. Peng. These are now presented correctly in this article.

The original article was corrected.

The online version of the original article can be found at https://oi.org/ $10.1007 / \mathrm{s} 00374-017-1235-8$

$\triangle$ X. H. Peng

xhpeng@issas.ac.cn

1 State Key Laboratory of Soil and Sustainable Agriculture, Institute of Soil Science, CAS, Nanjing 210008, China

2 IRD, UMR 242 iEES, Centre IRD Bondy, 32 Avenue H. Varagnat, 93143 Bondy, France

3 UAPV, UMR 'EMMAH', Site Agroparc, Cedex 09, 84914 Avignon, France

4 School of Agriculture and Biology, Shanghai Jiaotong University, Shanghai 200240, China

5 Indo-French Cell for Water Sciences (IFCWS), Department of Civil Engineering, Indian Institute of Science (IISc), Bangalore 560012, India 